\title{
Effect of reperfusion injury from distant ischemia to small intestine
}

\author{
Yefta Moenadjat, ${ }^{1}$ Aris Ramdhani, ${ }^{1}$ Wifanto Saditya Jeo, ${ }^{1}$ Wuryantoro Suharto, ${ }^{1}$ Retno Astri Werdhani ${ }^{2}$
}

pISSN: 0853-1773 • elSSN: 2252-8083 https://doi.org/10.13181/mji.v28i1.1468 Med J Indones. 2019;28:8-13

Received: June 15, 2016

Accepted: November 23, 2018

Author's affiliations:

${ }^{1}$ Department of Surgery, Faculty of Medicine, Universitas Indonesia, Cipto Mangunkusumo Hospital, Jakarta, Indonesia, ${ }^{2}$ Department of Community Medicine, Faculty of Medicine, Universitas Indonesia, Jakarta, Indonesia

\section{Corresponding author:}

Yefta Moenadjat

Department of Surgery, Faculty of

Medicine, Universitas Indonesia, Cipto Mangunkusumo Hospital,

Jalan Diponogoro No.71, Kenari, Senen, Central Jakarta 10310, DKI Jakarta, Indonesia

Tel/Fax: +62-21-3100050

E-mail: yefta.moenadjat@ui.ac.id

\begin{abstract}
BACKGROUND The ileum is the most vulnerable part of the small intestine that plays an important role as the motor of multisystem organ failure. Villous damage is demonstrated after ligation of supply artery in mice; however, there is no study on the ileum after distant ischemic organs. Thus, this study was aimed to find out ileal villous changes following reperfusion injury, the protective effects of ischemic hypothermia and ischemic preconditioning.
\end{abstract}

METHODS An experimental study conducted enrolled 21 subjects of Oryctolagus cuniculus. Ischemia is induced by ligation of the femoral artery for 4 hours. Eight hours after ligation was released, ileum and duodenal specimens were taken through laparotomy. H\&E stained specimens were examined for histomorphological changes. Villi change scores, tissue level of hypoxia-inducible factor-1a (HIF-1a), malondialdehyde (MDA), and occludin were statistically analyzed in four treatment groups, namely ischemia, ischemic hypothermia, ischemic preconditioning, and control.

RESULTS Intestinal villi changes were found following ischemic-induced arterial ligation. Ileal villi changes showed differences with the duodenum and controls as indicated by the villi damage scores, increased tissue HIF-1a and MDA, and decreased occludin levels. Ileal villi changes in the ischemic and ischemic hypothermia groups showed significant changes with controls; whereas the ischemic preconditioning group showed no significant differences.

CONCLUSIONS Ischemia at a distance leads to both histomorphological and biochemical damage of the ileal villi and disrupts the integrity of the intestinal mucosal barrier. In addition, the study showed a protective effect of ischemic preconditioning.

KEYWORDS ileal intestinal villi, ischemia, ischemia-reperfusion injury, ischemic hypothermia, ischemic preconditioning
Ischemia-reperfusion injury (I/RI) following hypoxia is known to be an issue leading to both cell injury and tissue/organ damage. Reactive oxygen species (ROS) produced in the mitochondria under hypoxia are injurious to living cells when released into the circulation, and distributed both locally and systemically. ${ }^{1,2}$ In reported studies on I/RI, ischemia is induced by the ligation of the arterial blood supply of a specific organ (namely, direct ligation); 3 thereby, noted histological damage after reestablished arterial flow is a reperfusion injury, but not as an impact of distant ischemia.
Intestinal failure is common following ischemia of any etiology, and hypoxemia due to hemorrhagic shock in multi-trauma patients or burn shock. The intestine is a visceral organ that is vulnerable to hypoxia due to local ischemia (acute intestinal ischemia) and/or distant ischemia. Among regions of the intestine, the ileum is the most vulnerable part. Ileal dysfunction (formerly known as ileus) manifests much earlier than any other part of the intestine; followed by multisystem organ dysfunction. In a critical setting, intestinal failure is attributed to the motor of multisystem organ dysfunction, the failure of 
which could be fatal. 4,5 Though there were elucidations of this nature, the evidence showed that inflammatory mediators released in hypoxia played an important role despite ROS. ${ }^{6}$

Many studies showed that the destructive effect of $\mathrm{I} / \mathrm{RI}$ is effectively attenuated through the application of ischemic hypothermia ${ }^{7}$ and ischemic preconditioning (IPC). ${ }^{8}$ The application of cold to the ischemic organ reduces metabolic activity thereby preventing ATP mismatch, and ultimately inhibits the acceleration of cell death. ${ }^{9}$ IPC is a technique that provides short repetitive episodes of ischemia to ensure that cells are adapted well before true hypoxia. IPC has been proven to minimize cell damage through the mechanisms of resistance to ischemia. ${ }^{10}$

So far, studies on the impact of reperfusion injury in the intestine remain scarce, and none intended to investigate ileal reperfusion injury due to distant ischemia. Thus, an experimental study on ileal villi damage was conducted following ischemia induced by ligating a large artery at a significant distance (in this case, the femoral artery) using Oryctolagus cuniculus as a model. The protective effects of both ischemic hypothermia and IPC were also the focus of the study.

\section{METHODS}

An experimental study conducted in an animal laboratory of the Institut Pertanian Bogor (IPB) enrolled 21 subjects of New Zealand White rabbits (Oryctolagus cuniculus) according to the Federer formula, with regard to the $3-R$ principles in the use of animals for experimental study. The study focused on three different treatment groups, i.e., ischemia, ischemic hypothermia, and IPC in addition to control. An in vivo model was used to avoid encountering ethical issues when using human subjects. The rabbit was chosen as it met the criteria of bioavailability on the intestinal study.11 The ileum was chosen because it is the part of the intestine that is most vulnerable to hypoxia. In addition to the ileum, specimens of the duodenum in the same subject were taken for comparison purposes.

In preparation, subjects were adapted for seven days before the study, fed, and were scrutinized. Ischemia was induced by ligating the left femoral artery through a $5 \mathrm{~cm}$ long incision in the inguinal region under general anesthesia using intravenous ketamine. Ischemia was confirmed by monitoring oxygen saturation. The ligation was maintained for 4 hours. Ischemia hypertension was induced by applying a cold pack around the affected limb, wrapping it with aluminum foil, and keeping it maintained for 4 hours after ligation. The temperature was maintained for $21-24^{\circ} \mathrm{C}$. IPC was carried out by repetitive ligation and release every $10 \mathrm{~min}$, respectively, and was applied in three cycles. ${ }^{12}$ The control group consisted of animals that did not receive treatment but were biopsied. After ligation, subjects were set free in their cages for 8 hours. Within 8 hours of reperfusion, specimens of the ileum and the duodenum were taken through a laparotomy. Subjects were sacrificed following regulations applied in the animal laboratory of the IPB. Specimens were placed in sterile tubes and subjected to analyses.

\section{Study on histomorphologic changes in villi using hematoxylin and eosin (H\&E)}

Specimens were prepared for sectioning of paraffin-embedded tissue and staining with H\&E. Histomorphologic changes in the villi were investigated under the light microscope with objective lens magnifications of 10, 40, and 100 times, respectively. A scoring system was used to represent villi changes in accordance with the Chiu/Park scale, ${ }^{13}$ where a score of 0 referred to normal mucosa, 1 represented subepithelial vacuolization, 2 represented extension of subepithelial space with moderate filling, 3 represented severe lifting down to the base of the villi, 4 represented denudation of the villi ends, 5 represented denudation of the villi with a dilated capillary, 6 represented disintegrated lamina propria, 7 represented a crypts injury, 8 represented an infarcted mucosa, and 9 represented infarct of transmural. Interpretations were performed by an animal pathologist in the laboratory of animal pathology, the IPB.

\section{Study on hypoxia and oxidative stress}

Tissue hypoxia-inducible factor-1a (HIF-1a) and malondialdehyde (MDA) were investigated to uncover evidence of mucosal hypoxia and oxidative stress. The study was conducted in the biochemical laboratory of the Department of Biochemistry, Faculty of Medicine, Universitas Indonesia.

\section{Study on HIF-1a}

Rabbit HIF-1a ELISA Kit (Elabscience) was used per the protocol provided in the manual. The optical density value was measured in a microplate reader representing the level of tissue HIF-1a ( $\mathrm{ng} / \mathrm{ml})$. The procedure was performed in duplicate. 


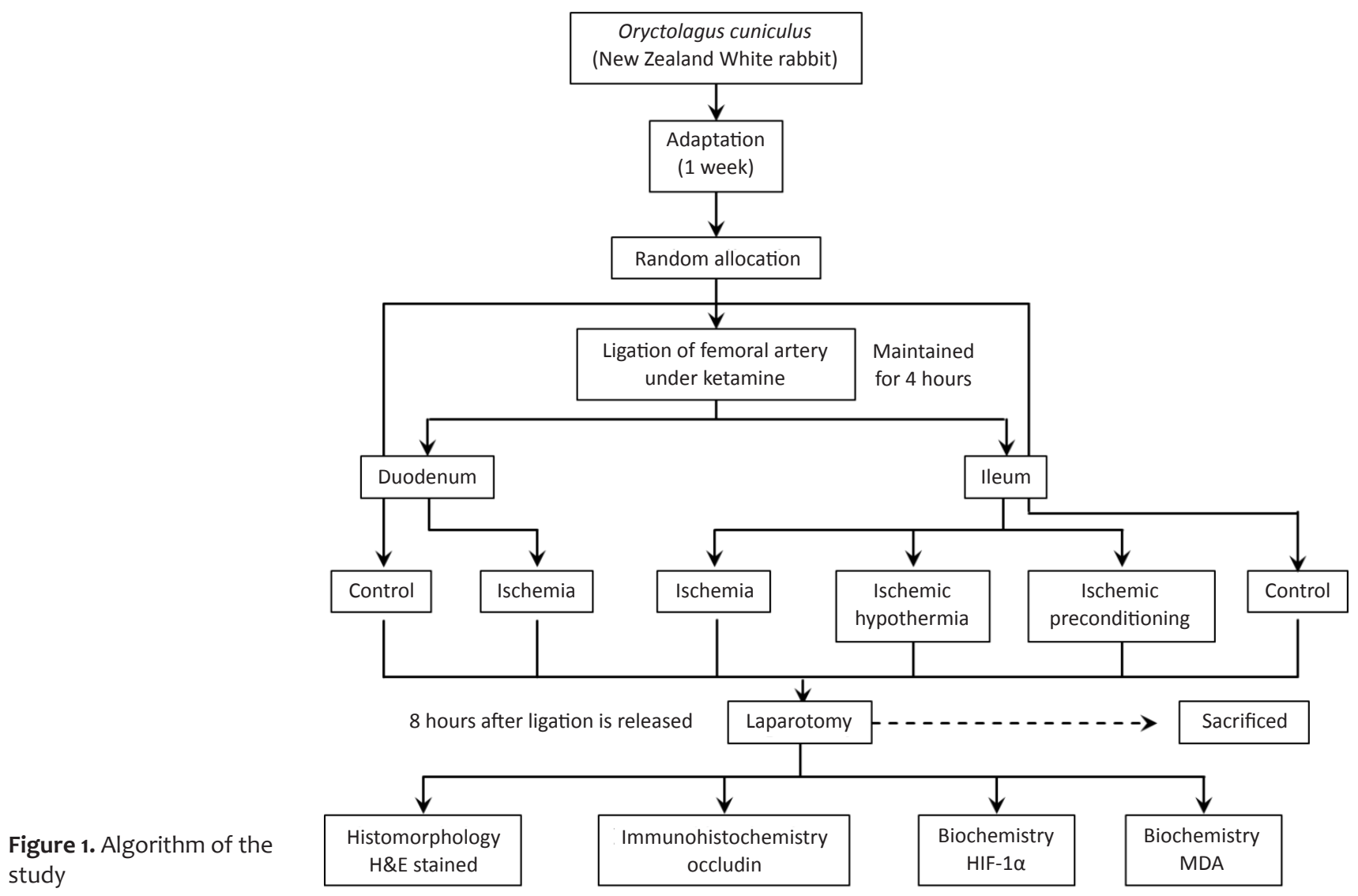

\section{Oxidative stress}

Tissue MDA as a marker of lipid peroxidation reflecting oxidative stress was the focus of the study. The value of thiobarbituric acid, which is directly proportional to the value of peroxide, was measured (in $\mathrm{ng} / \mathrm{ml}$ ) in accordance with Will's method. The procedure was performed in duplicate.

\section{Study on mucosal barrier integrity}

Occludin at the tight junction indicates that the integrity of the ileal mucosa was subjected to investigation; This aspect sought to find biochemical evidence of the disintegrated epithelial lining of the intestinal mucosa. A rabbit OCLN enzyme-linked immunosorbent assay (ELISA) kit (Elabscience) was used for this purpose. The occludin level was measured $(\mathrm{ng} / \mathrm{ml})$ in duplicate.

All variables were subjected to statistical analysis using statistical package for the social sciences (SPSS) version 20. A p-value of less than 0.05 was considered to be statistically significant. The Ethics Committee of the Faculty of Medicine Universitas Indonesia approved the study (No 495B/H2.F1/ETIK/2014). (Figure 1)

\section{RESULTS}

Of 21 subjects, 20 were analyzed; a subject was excluded as it was found dead during laparotomy for biopsy. After ligation, arterial oxygen saturation $\left(\mathrm{SaO}_{2}\right)$ in ischemic limbs ranged from $62-72 \%$ and mean (SD) $67(3.95 \%)$, while on the contralateral side it ranged from $97-99 \%$ and mean (SD) 98.28 (1.03\%). After ligation was released, the $\mathrm{SaO}_{2}$ in ischemic limbs ranged from 97-100\% and mean (SD) 98.42 (0.97\%), demonstrating that perfusion was restored (Figure 2).

Histomorphological changes in the villi of the ischemic duodenum and the ileum were found to vary. Flattening of the villi was found in all specimens. Vacuolization was the characteristic feature found in the ischemic group but was minimal in the hypothermia as well as IPC groups (Figure 2).

Using the Mann-Whitney test, the scores of villi damage in the ischemic duodenum (Figure 3) were higher when compared with control $(p<0.004)$, the ischemic ileum was higher when compared with control $(p<0.001)$, and the difference between the ischemic ileum and the ischemic duodenum was significant $(p<0.001)$. 

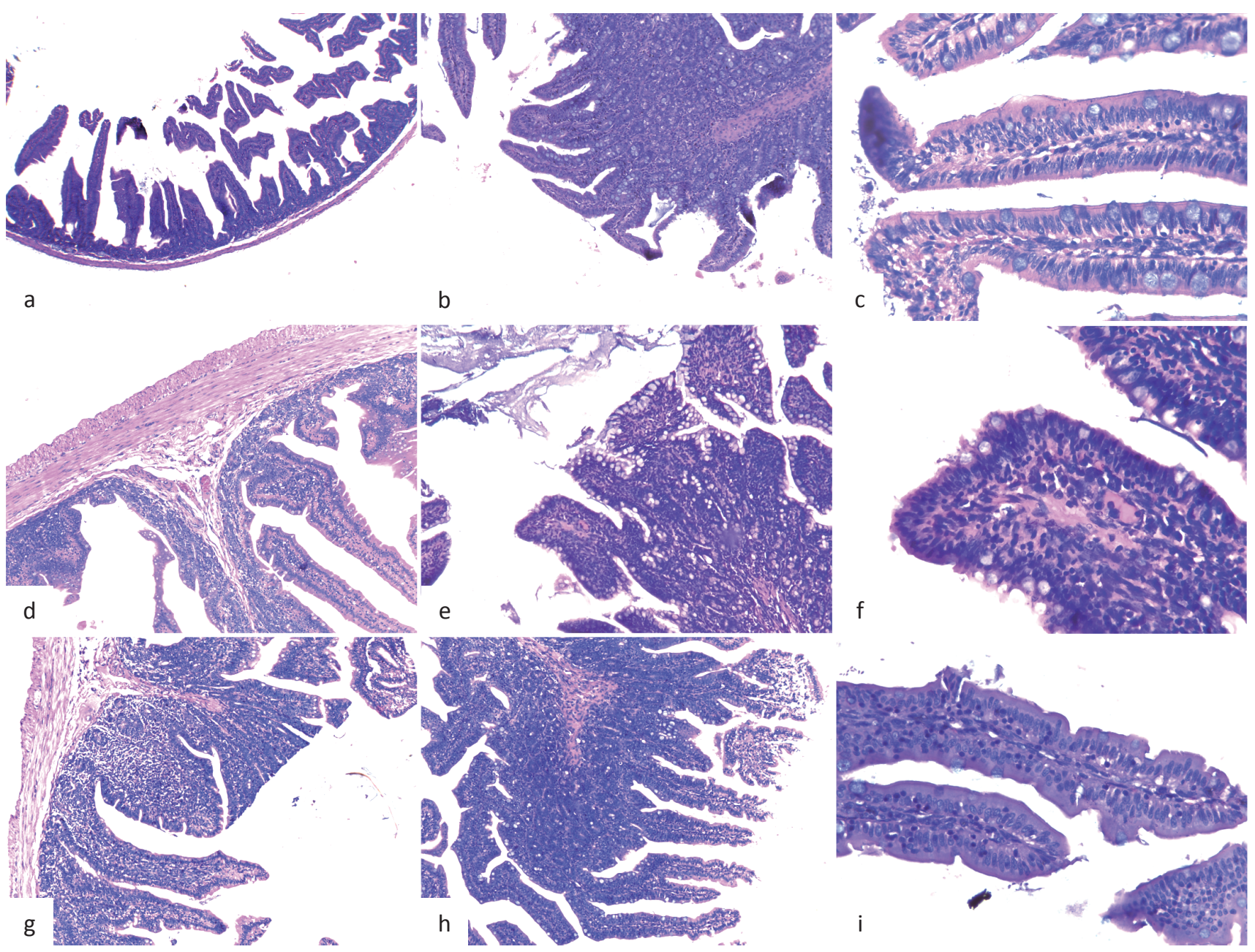

Figure 2. Ileal villi histomorphologic changes on ischemic reperfusion injury in H\&E stained samples: (a) Shortening and flattening villi of ischemic group under $40 \mathrm{x}$ magnification; (b) Flattening villi is clearly shown under magnification of $100 \mathrm{x}$; (c) Vacuolization of villi under magnification of $400 \mathrm{x}$; (d) Histomorphologic changes in preconditioning group referred to relatively minimal as seen under magnification of $40 \mathrm{x}$; (e) Even though villi changes in ischemia referred to minimal, vacuolization is clearly shown under magnification of $100 \mathrm{x}$ and $400 \mathrm{x}$; (f) and (g) Histomorphologic changes in ischemic hypothermia. Relatively normal villi are shown: (h) In relatively normal villi found in ischemic hypothermia group, distribution of acute inflammatory cells is clearly shown under magnification of $100 \mathrm{x}$. (i) In this ischemic hypothermia, vacuolization is minimal as shown under magnification of $400 \mathrm{x}$

In the ileum, damage to the villi was found to be higher in the ischemic ileum, whereas in both the ischemic hypothermia and the IPC groups, a lesser degree of damage was found. In the ischemic group, the tissue HIF-1a in both the duodenum and the ileum increased, and the highest levels were found in the ischemic ileum. In both the ischemic hypothermia and the IPC groups, tissue HIF-1a was found to have lower significance.

Tissue MDA increased in both the duodenum and the ileum and was highest in the ischemic ileum. In both the ischemic hypothermia and the IPC groups, tissue HIF-1a was found to have lower significance. The occludin level decreased in the ileum of the ischemic group and showed the least in both the ischemic hypothermia and the IPC groups. Analyses showing differences in villi scoring, MDA,
HIF-1a, and occludin were significant under all conditions. In addition, Bonferroni post-hoc tests were performed on HIF-1 $1 \alpha$ and occludin showing $p<0.001$ (Figure 3 ).

\section{DISCUSSION}

Changes in intestinal villi were shown in the study following ischemic-induced arterial ligation, which is a reperfusion injury following ischemia at a significant anatomical distance. Changes in the villi were noted in both the ileum and the duodenum. However, to date, there is no consensus about villi damage, the definition, criteria, and classification. ${ }^{14}$ Some studies prefer to use "villi atrophy" to describe villi damage, while others prefer to use "villi flattening" to convey the significant decrease in crypt height. 

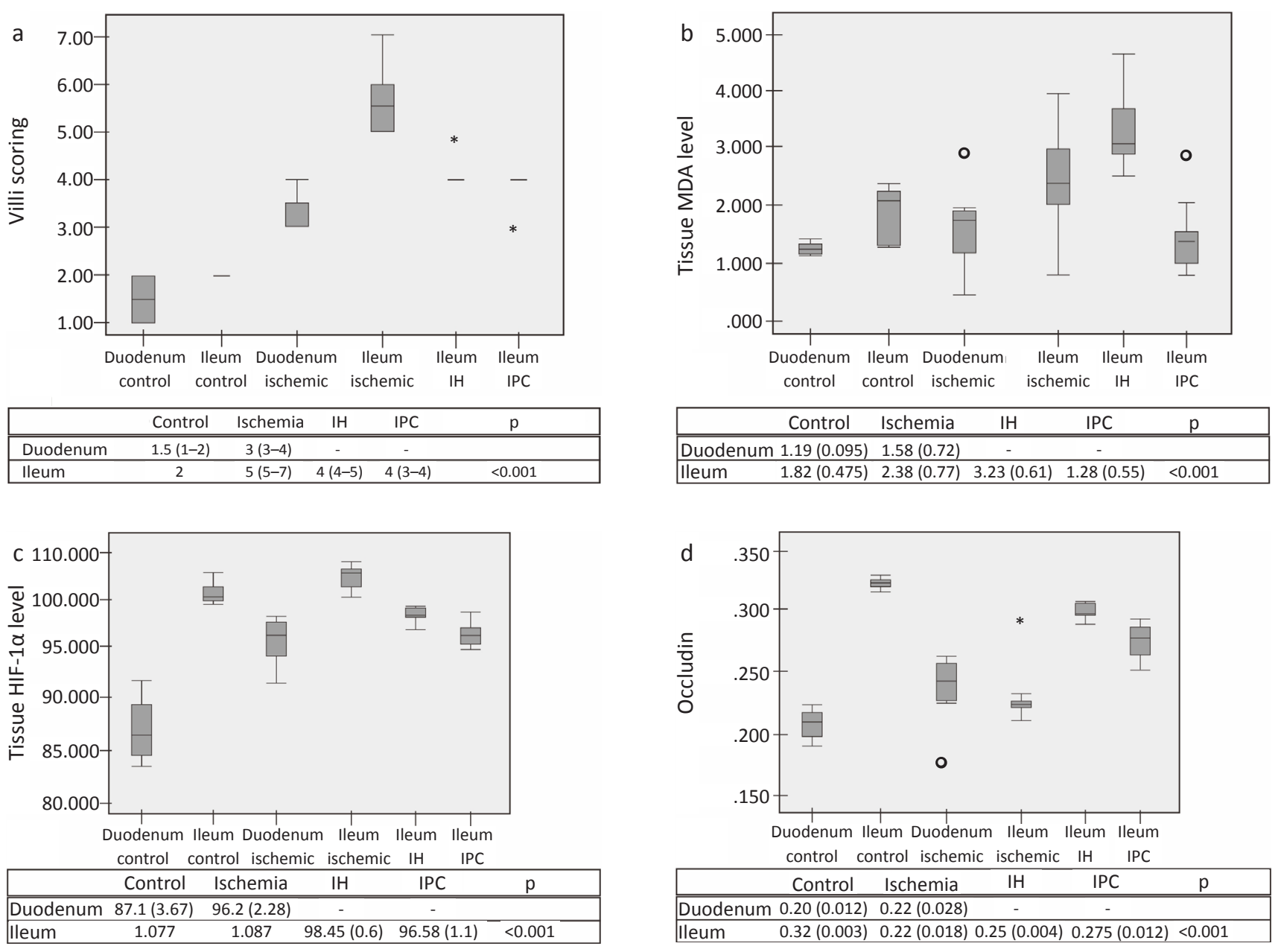

Figure 3. Effects of ischemia/reperfusion injury on ileal villi (data below graphic are presented in mean (SD) or median (min-max)): (a) Scores of villi damage; (b) Level of tissue HIF-1a; (c) Level of tissue MDA; and (d) Occludin in ischemia, ischemic hypothermia, and IPC. There were significant differences between ischemia, ischemic hypothermia, and IPC to control $(p<0.001)$. IH=ischemic hypothermia; IPC=ischemic preconditioning; MDA=malondialdehyde; HIF-1a=hypoxia-inducible factor-1 $\alpha$

In this study, the scoring system used was in accordance with the Chiu/Park scale. ${ }^{3}$ If the study just focused on villi flattening, then no analysis could be done particularly to determine the differences between intestinal segments since all the specimens indicated this kind of pathology, including the control. Changes in the villi including flattening, detached villi ends, crowded vacuoles in the villi stroma, congested villi, and subepithelial lifting are features that represent a reperfusion injury as the effect of distant ischemia. As hypothesized, a change in the ileal villi is more severe than that in the duodenal. This is reasonable since the duodenum is well vascularized because of its anatomical function. It is supplied by at least nine branches of the gastroduodenal artery that enable the duodenum to adapt better to hypoxia than the ileum.

In contrast, the ileum is supplied by multiple arcades (short terminal branches) arising from the superior mesenteric artery in addition to having a thinner wall (less plica circular), rich with numerous aggregated nodules of lymphoid tissue (Peyer's patches). Somehow, such a condition has a predilection of ROS accumulation, bacterial stasis, as well as inflammatory mediators. The study shows that the ileum is more vulnerable than the duodenum, in contrast to studies indicating that the duodenum is the most vulnerable.

A surrogate marker of hypoxia, namely HIF-1a, which was found to be increased, indicating hypoxia of the villi as the study focused on the tissue level instead of the serum level. ${ }^{15}$ The condition applied to increased tissue level of MDA, a marker of lipid peroxidation, indicating oxidative-stressed villi. After 8 hours or reperfusion, changes in the villi such as flattening and detached villi ends are features indicating villi apoptosis, ${ }^{16,17}$ even though apoptosis needs to be proven further. The conditions suggesting an irreversible change are shown in an earlier study by Guan et al..$^{18}$ These histomorphologic 
changes indicating epithelial lining integrity are no longer maintained, in other words, the mucosal barrier is disrupted. The occludin level is decreased indicating disassembled epithelial cell-to-cell tight junctions.

In this study, IPC protected changes in ileal villi effectively, as is shown in previous studies in rat intestinal mucosa $\mathrm{a}^{19,20}$ and myocardia. ${ }^{21}$ The temperature applied in ischemic hypothermia was between $21^{\circ} \mathrm{C}$ and $24^{\circ} \mathrm{C}$ using ice wrapped around the ischemic limb. This method is effective to use in the study of therapeutic hypothermia when applying the temperature of $13^{\circ} \mathrm{C}^{22}$ This might be of benefit in a clinical setting.

Even though the study enrolled a small number of subjects, ${ }^{23}$ the differences of each treatment group is shown. Rather than rodents, rabbit intestine represents those in primates as well as humans, even though rabbits have a different dietary pattern, 23,24 more studies in humans are required. This is the limitation of a study. Somehow, the novel nature of this study was that it differed from its predecessors. First, this in vivo study focused on the impact of remote I/RI. Second, this study enrolled rabbits whereas others enrolled mice. Third, the ischemia was induced by ligation of the femoral artery whereas others preceded direct ligation of a definite artery. Fourth, the study focused on the ileum whereas others on the jejunum. Fifth, this study showed that the ileum is the part of the intestine with the most susceptible to hypoxia.

Through this study, it was concluded that distant ischemia leads to intestinal reperfusion injury. Hypoxic and oxidative-stressed villi are followed by disruption of both intestinal integrity and the mucosal barrier. This study showed that both IPC and ischemic hypothermia have a protective effect against reperfusion injury at a distance.

\section{Conflicts of Interest}

Authors affirm no conflict of interest.

\section{Acknowledgment}

This work was supported by the Operational Research's Grant of Cipto Mangunkusumo Hospital 2015.

\section{Funding Sources}

This work was funded by the Operational Research's Grant of Cipto Mangunkusumo Hospital 2015.

\section{REFERENCES}

1. Carden DL, Granger DN. Pathophysiology of ischemiareperfusion injury. J Pathol. 2000;190(3):255-66.

2. Collard CD, Gelman S. Pathophysiology, clinical manifestations, and prevention of ischemia-reperfusion Injury. Anesthesiology. 2001;94(6):1133-8.
3. Eltzschig HK, Collard CD. Vascular ischaemia and reperfusion injury. Br Med Bull. 2004;70:71-86.

4. Leaphart CL, Tepas JJ. The gut is a motor of organ system dysfunction. Surgery. 2007;141(5):563-9.

5. Sertaridou E, Papaioannou V, Kolios G, Pneumatikos I. Gut failure in critical care: old school versus new school. Ann Gastroenterol. 2015;28(3):309-22.

6. Schofield Z, Woodruff TM, Halai R, Wu MC, Cooper MA. Neutrophils-a key component of ischemia-reperfusion injury. Shock. 2013;40(6):463-70.

7. Zhu J, Fu Y, Jiang J, Liang JK, Huang ZT. Therapeutic hypothermia reduces intestinal ischemia/reperfusion injury after cardiac arrest in rats. Afr Journal Biothecnol. 2011;10(38):7497-502.

8. Zhang B, Zhao Y, Bao L, Wang LH, Guo HB, Zhang WX, et al. Protective effects of remote limb preconditioning on ischemia reperfusion injury in rats liver. J Gastroenterol Hepatol Res. 2013;2(9):791-4.

9. Moore EM, Nichol AD, Bernard SA, Bellomo R. Therapeutic hypothermia: benefits, mechanisms and potential clinical applications in neurological, cardiac and kidney injury. Injury. 2011;42(9):843-54.

10. Erling Junior N, Montero EF, Sannomiya P, Poli-de-Figueiredo LF. Local and remote ischemic preconditioning protect against intestinal ischemic/reperfusion injury after supraceliac aortic clamping. Clinics (Sao Paulo). 2013;68(12):1548-54.

11. Weis CP, LaVelle JM. Characteristics to consider when choosing an animal model for the study of lead bioavailability. Chem Speciat Bioavailab. 1991;3(3-4):113-9.

12. Zhang Y, Wu YX, Hao YB, Dun Y, Yang SP. Role of endogenous opioid peptides in protection of ischemic preconditioning in rat small intestine. Life Sci. 2001;68(9):1013-9.

13. Oltean $\mathrm{M}$, Olausson $\mathrm{M}$. The Chiu/Park scale for grading intestinal ischemia-reperfusion: if it ain't broke don't fix it! Intensive Care Med. 2010;36(6):1095.

14. Elli L, Zini E, Tomba C, Bardella MT, Bosari S, Conte D, et al. Histological evaluation of duodenal biopsies from coeliac patients: the need for different grading criteria during followup. BMC Gastroenterol. 2015;15:133.

15. Semenza GL. Hydroxylation of HIF-1: oxygen sensing at the molecular level. Physiology. 2004;19(4):176-82.

16. Ikeda H, Suzuki $Y$, Suzuki M, Koike M, Tamura J, Tong J, et al. Apoptosis is a major mode of cell death caused by ischaemia and ischaemia/reperfusion injury to the rat intestinal epithelium. Gut. 1998;42(4):530-7.

17. Kannan K, Jain SK. Oxidative stress and apoptosis. Pathophysiology. 2000;7(3):153-63.

18. Guan Y, Worrell RT, Pritts TA, Montrose MH. Intestinal ischemiareperfusion injury: reversible and irreversible damage imaged in vivo. Am J Physiol Gastrointest Liver Physiol. 2009;297(1):G187-96.

19. Moore-Olufemi SD, Olufemi SE, Lott S, Sato N, Kozar RA, Moore FA, et al. Intestinal ischemic preconditioning after ischemia/ reperfusion injury in rat intestine: profiling global gene expression patterns. Dig Dis Sci. 2010;55(7):1866-77.

20. Brzozowski T, Konturek PC, Konturek SJ Pajdo R, Kwiecien S, Pawlik M, et al. Ischemic preconditioning of remote organs attenuates gastric ischemia-reperfusion injury through involvement of prostaglandins and sensory nerves. Eur $J$ Pharmacol. 2004;499(1-2):201-13.

21. Tyagi P, Tayal G. Ischemic preconditioning of myocardium. Acta Pharmacol Sin. 2002;23(10):865-70.

22. Shah TA, Mauriello CT, Hair PS, Sandhu A, Stolz MP, Bass WT, et al. Clinical hypothermia temperatures increase complement activation and cell destruction via the classical pathway. J Transl Med. 2014;12:181.

23. Graham ML, Prescott MJ. The multifactorial role of the $3 R s$ in shifting the harm-benefit analysis in animal models of disease. Eur J Pharmacol. 2015;759:19-29.

24. Mapara M, Thomas BS, Bhat KM. Rabbit as an animal model for experimental research. Dent Res J (Isfahan). 2012;9(1):111-8. 